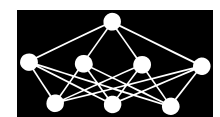

\title{
DYNAMICS OF SINUSOIDAL ALPHA WAVES ASYMMETRY IN BRAIN ELECTRICAL FIELD
}

\author{
J. Faber*ł M. Novák, M. Kovaljov, R. Chalupováł, P. Bouchner*
}

\begin{abstract}
There is no methodical approach suitable for definition of the periodical or non-periodical, stationary or nonstationary curves of brain signals with a help of amplitude, frequency, phase etc. values. It is difficult to determinate the wave shape, i.e. the problem is how to solve the respective pattern recognition. Therefore, we tried to propose a simple method for praxis by help of measurement two main wave time components, interpreting a sinusoidal alpha wave as a triangle, where there is an anterior and a posterior part of wave ascending and descending abscissas in a hope that the sufficient measure are presented by the "legs" only or distances between upper and bottom peak of the wave. All the values of total ascendants are divided by all values of total descendants. For the method validity estimation it was made for this computation separately in two different psychical states - the relaxation and the calculation activity, both with eyes closed. Results are presented as quotient (quotus alpha) which means alpha waves symmetry. If the quotient is equal to 1 , or is near to 1 , is the alpha wave full or almost symmetrical. When the quotient is lower than 1 the ascendant is shorter than descendent, then alpha wave is asymmetric and has inclination to the left side. In contrary if the quotient is higher than 1 the ascendant is longer than descendent, alpha wave is again asymmetrical, but inclination is oriented to the right side. During mentation is usually quotient lower one and the ascendant is still more lover, alpha waves are sheer, the inclination to the left is more expressive.
\end{abstract}

Key words: EEG, electrical brain signals, basic alpha rhythm, alpha pattern recognition, alpha amplitude, alpha steepness, alpha waves asymmetry, reinforced thinking, uncertain factors

Received: September 13, 2019

DOI: $10.14311 /$ NNW.2019.29.026

Revised and accepted: December 30, 2019

\section{Introduction}

Electrical field in the brain was recorded by noninvasive way first already by Johannes Berger in 1924 and named by him as elektrenkephalogram (now electroencephalogram, EEG) [7]. This phenomenon was generated by synchronous electrical

*J. Faber, M. Novák - corresponding author, M. Kovaljov, P. Bouchner; Czech Technical University in Prague Faculty of Transportation Sciences, K616 Konviktská 20, Prague 1, 11000 Prague 1, E-mail: mirko@fd.cvut.cz

${ }^{\dagger}$ J. Faber, R. Chalupová; Institute of Biofeedback and EEG diagnostic, Prague 
potentials of neurons in thalami cortical reverberation iterative system (TCRIS). The feature of these potentials remind the trajectory of the most complex system and is composed of regular (harmonic) and irregular (random) variables. From certain point it reminds a noise. Discussion about this topic was referenced in many works, e.g. in the $[5,25-27,31,34,36]$.

The basic electrical brain rhythm of health, waked, fully relaxed human which can be recorded on the scalp is pseudo rhythmic, pseudo periodic and consists of alpha and beta activity of sinusoidal feature of frequencies $8-13 \mathrm{~Hz}$, eventually $14-30 \mathrm{~Hz}$ of the amplitude mostly $20-100$ microvolt.

The highest alpha amplitude showed the Yoga mediation [1], then follows all the time decreases during relaxed state with eyes closed, next is mentation with eyes closed, then quiet state with eyes open and finally minimal alpha amplitude is present during mentation with eyes open. In the EEG curves was high content of noise, e.g. during vigilance increases on average to $20-30 \%$ and during sleep and epileptic activity still decreases [18].

Because the typical human brain contains approximately 100 milliards (billions) neurons, one cannot believe from quantitative point of view that the brain can be a deterministic system. Moreover, every neuron has about 10 thousand of synaptic (interface) contacts, which mediate information acquisitions from other neurons. Every synapsis has different weight, different neuronal impulse sequences (NSI) and different qualitative properties (excitation or inhibition). It is not possible, to compute together all these factors and concentrate them in simple sinusoidal alpha wave by simple way. All these principal properties, inclusively the physiological and pathological automatic or semiautomatic behavior, lead us to considerations about subconscious thinking, which occurs in sleep but during vigilance as well $[4,5,8,9,11-15,17,18,21,24,26,27,29]$.

Our previous experiments showed interesting alpha changes during mentation [15] photo stimulation, hyperventilation and intrapsychic tension e.g. during traveling in tunnel $[11,33]$. Therefore, our temptation was to propose new simple analytical view for alpha waves morphology.

\section{Experimental data and analytical method}

The research was made on cohort consisted of 22 subjects, aged 7 to 48 (18 men, 4 women). From this group were 15 people aged 10 to 48 years: 2 boys suffered from ADHD (attention deficit/hyperactivity disorders) with typical EEG abnormality, 3 women (one woman of age 23 years, suffered from anxious phobic syndrome with diffusely abnormal EEG, 2 women being without difficulties, aged 31 and 34 years, with normal EEG curves), 10 men, aged from 22 to 48 years, (but one healthy man who was 48 years old and the man aged 33 years suffered from compensated posttraumatic epileptic syndrome), remaining 8 men aged 22 to 33 years were without subjective and objective difficulties, high school educated, (4 man had quite abnormal EEG), over average intelligence, controlled by Raven test. All these 10 probands had "correct" alpha waves asymmetry with inclination to the left side (see later) and no faults in two digit numbers addition. Above mentioned 15 subjects had good calculation with two exceptions only: a phobic girl aged 23 years and boy aged 10 years with ADHD syndrome, both with abnormal EEG 
Faber J. et al: Dynamics of sinusoidal alpha waves asymmetry in brain...

curves. The remaining set of 7 people aged 7 to 30 years had abnormal EEG (with one exception, man aged 26 years), non-typical alpha waves asymmetry with inclination to the right side, 4 people aged 7 to 18 years had ADHD syndrome, 3 people aged 9 to 25 years made faults during calculation. It was summarized up, that 15 subjects from 22 had not alpha waves precise sinusoidal but on the contrary of asymmetrical shape with steeper and shorter ascendants and longer descendants part of alpha wave.

The remaining set of 7 people aged 7 to 30 years had abnormal EEG (with one exception, healthy man aged 26 years), non-standard alpha waves asymmetry with inclination to the right side, 4 people aged 7 to 18 years had ADHD syndrome, 3 people aged 9 to 25 years made faults during calculation.

Standard 19 channels EEG device (DEYMED firm, Czech Republic) for scalp EEG curves recording was used for many psychological tests, eyes open: OCULI APERTI, ACAP, OO, eyes closed: OCULI CLAUSI, OCCL, ZO, PAX, REALAXATION, abbreviated Raven test (RAV), hyperventilation through nose (HVN), hyperventilation through mouth (HVO), reading, addition of one- (S.) or two digit (C.) numbers: CALCULATION, CAL.S., or CAL.C., with good results B ("bene"), or bad results $\mathrm{M}$ (male, mistake), photo stimulation (FS), eventually other tests. The recording took 25 to 35 minutes. In this study were used two states only, relaxation (PAX) and mentation with addition of one digit (CAL.S.) for children or two digits (CAL.C.) for adults, all with eyes closed.

Because of limited time for measurement was selected only one EEG channel usually from temporoparietooccipital region. For both states was taken the same channel. However, for an eventual complete automation it was prepared a computer program suitable for analysis of states existing at the channels at which can be many different psychic states.

Next details of method are described in Fig. 1 and Fig. 2, which are discussed also later in respective paragraph. The respective EEG curves are described both by visual way and by Gabor's mathematical analysis, i.e. by the power spectrum. The special regards are given to the results of psychotests and simultaneous occurrence of alpha waves, as concerns the frequency and amplitude, and on brain electrical activity mapped during ascendant (ALAS) and descendent (ALDE) part of fusiform alpha wave configuration.

\section{Discussion on obtained results}

At first was investigated the modestly large proband team of 22 persons, 18 men and 4 women. To this were added children aged 7-18 years suffered from ADHD syndrome: 5 boys with abnormal and for ADHD typical EEG and one girl aged 11 years with mild abnormal EEG and quotient of alpha steepness (quotus alpha) 0.96 in PAX and 1.22 during non-correct calculation (CAL.M.), 12 years old boy had quotient in PAX 0.83, in good calculation (CAL.B.) 0.81 which is small difference (0.02), but typical for "good working" brain. Two next boys had good calculation but non typical inverted (0.80 PAX, 0.82 calculation) or equalized quotient (1.09 PAX and 1.09 CAL.B.). 2 other boys had fault calculation and atypical tendency to the right sloping and inverted quotients (1.07 PAX, 0.73 CAL.M., and 0.91 PAX, 1.11 CAL.M.). Alpha descendent elongation during good and successful mentation 


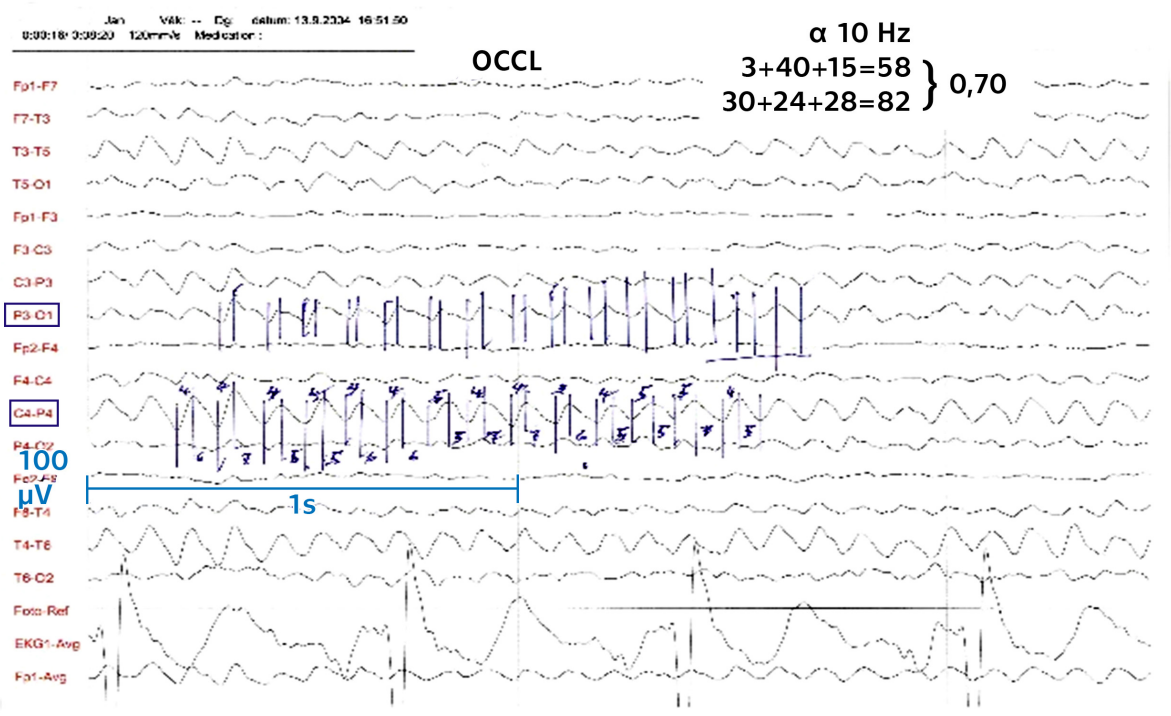

Fig. 1 Graphical method shows description of alpha waves shape in the healthy probands, aged 28 years. The standard EEG curve was printed in expanded form four times longer and 3 times higher than habitually. Short lines of abscissas were made parallel to ordinate (y-axis). These abscissas are perpendicular to $x$-axis and intersect the negative and positive alpha waves peaks. In the other words every alpha wave is designated after every half period named $\pi$. By this way is designated ascending and descending alpha wave parts and so one can measure the inter distance between them. This process was realized in EEG section with length 10-15 alpha waves during relaxation (PAX) and calculation (CAL). All values total of ascendants are divided by all values total of descendant separately for both states - relaxation and calculation. Result is presented as quotient (quotus alpha) which represents alpha waves symmetry. If the quotient is equal to 1 or is near to 1 is the alpha wave full symmetric or close to symmetry. When the quotient is lower than 1 then is shorter the ascendant than the descendent, then the alpha wave is more asymmetric and has bigger inclination to the left side. Moreover, contrary if the quotient is higher than 1 the ascendant is longer than descendent alpha wave is again asymmetrical, but inclination is oriented to the right side. During mentation quotient is usually lower and the ascendant is shorter, the inclination to the left is more expressive. The last curve is also expanded electrocardiogram (ECG).

is due to increased beta activity, which is perceptible optically as well. Alpha ascendant shortening is perhaps due to numerous enhancement neuronal impulses in TCRIS.

From the 16 subjects, aged 22-48 years, 9 men and 1 woman aged 24 to 33 years, were neither subjective nor objective disturbances (except one posttraumatic epileptic with subclinical typical paroxysmal spike and wave graph-elements in EEG), good reckoners, all with typical left side sloping alpha waves and quotients on an average 0.87 PAX and 0.83 CAL.C.B., 4 people had mild diffusively abnormal EEG. 
Faber J. et al: Dynamics of sinusoidal alpha waves asymmetry in brain...

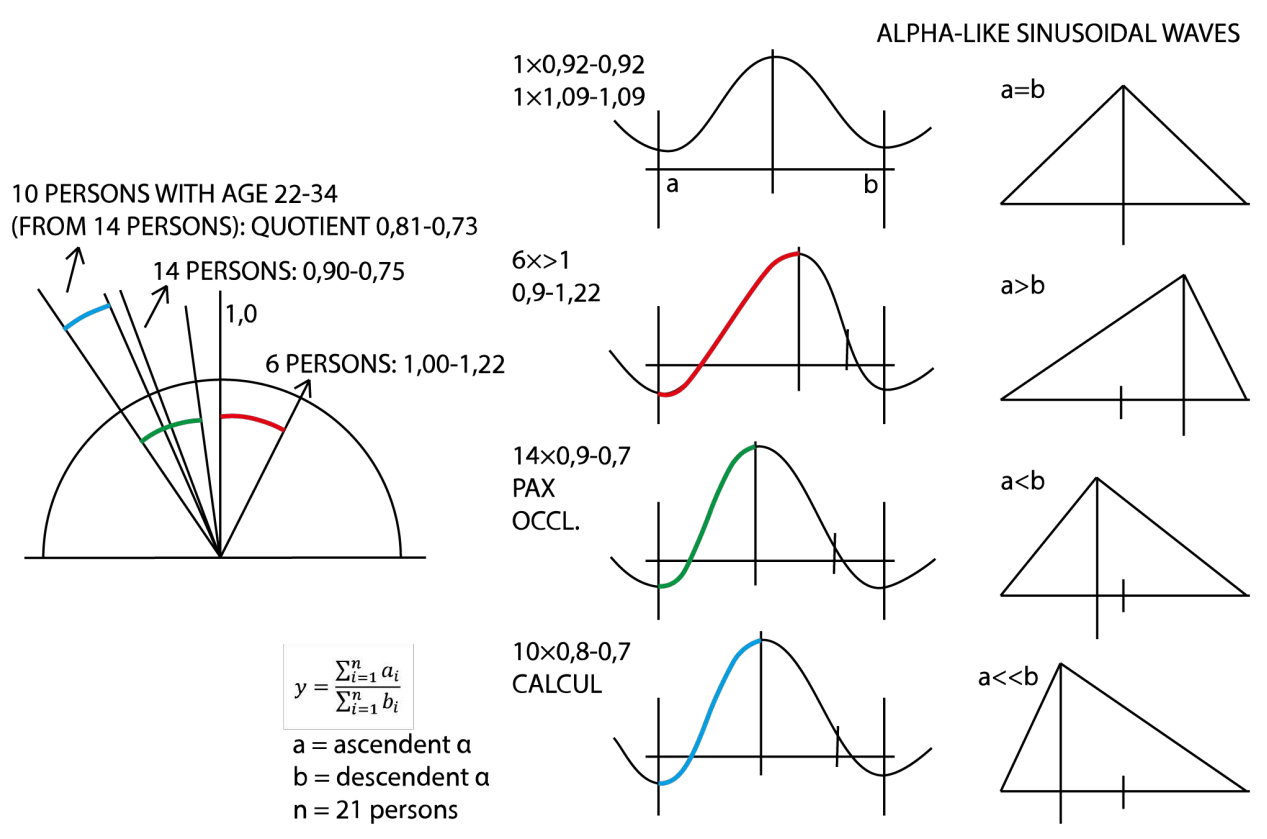

Fig. 2 On the right side is shown the alpha waves analysis (quotus alpha). Following process was applied to quiet (relaxing state) and activated (calculation) with EEG curve accompanying. Both states, relaxation and calculation during eyes closed had in EEG curves alpha activity. For objectivity and simplicity were used triangles instead of the alpha waves. Scheme shows four important specimens of alpha waves shape (from up to down): 1. symmetrical triangle, where abscissa on $x$-axis a equals $b$ and also alpha wave on the left is symmetrical (10\% subjects from our probands), 2. triangle with the longer abscissa a than $b$ represent asymmetrical alpha wave inclined to the right side, (30\% people, usually with non-correct calculation), 3. possibility is triangle with shorter abscissa a than $b$ which represent again asymmetrical alpha, but with inclination to the left side (60\% of our probands with correct calculation), 4. The last possibility is similar to the case 3, but with extreme asymmetry where is more wave much more inclined to the left side during correct calculation test. On the left hand there is illustration of this problem with $a$ and $b$ abscissas in semicircle, which makes principle of quotus alpha more clear. You can see the equation bottom right. Quotient does not have goniometric function, but it only represents legs in nonrectangular triangle, they are not grades in angles, but values ratios only. (one angle grade "equals" 1, i.e. 1 unit of quotus alpha). Average of quotients in 15 subjects during PAX is 0.90 and 0.75 during calculation, only 2 subjects did mistakes during calculation and 4 subjects had quotient about 1, therefore difference is 0.15 . Ten intelligent young probands from above 14 presented people had average quotient 0.81 in $P A X$ and 0.71 during calculation CAL.B., therefore difference is 0.10 (quotus alpha less than 1). Average quotient in 7 subjects, inclusive 4 ADHD syndrome (from all 22 people) with varied results was 0.89 during $P A X$ and 0.98 during calculation, therefore difference is not only small (0.09), but also inverted (quotus alpha higher than 1). Average quotient in all 22 people in PAX were 0.89 and during calculation 0.86 (CAL.B. and M.), therefore a little difference 0.03 . 
From the quoted 6 children (with ADHD) and next 6 adolescents subjects aged 23-25 years, had "neurotic" symptoms, incorrect calculation, quotients inclined to right side (on an average 0.89 PAX, 0.98 CAL.M.) and in 4 people were inverted quotients (on an average 1.21 PAX, 1.15 CAL.M.).

These 12 people had in EEG spectrum during calculation 8 time dichotomy alpha activity, e.g. during quiet state is in alpha frequency band only one dominant frequency (e.g. $9 \mathrm{~Hz}$ ), but during mental activity appeared two peaks (e.g. 9 and $10 \mathrm{~Hz}$ ). From this number 6 adolescent people 2 women suffered from anxiophobic syndrome: 23 aged (BAA), bad reckoner, EEG expressively abnormal but quotients were typically inclined to left side (0.92 PAX, 0.80 CAL.M.), next woman aged 34, good reckoner, normal EEG but quotient in PAX was inclined atypically to nontypical left side (1.42 PAX, 0.84 CAL.B.). The alpha activity dichotomy is to be see very often in "neurotic" subjects, perhaps due to intrapsychic tension. Alpha waves are steeper in healthy subjects ( 8 times in 13 men and 1 woman) and 2 times only in 10 subjects with incorrect calculation. These results are in agreement with our previous experiences.

The frequency of alpha activity acceleration in the course of calculation or generally in the course of mental activity was $0.5-1.5 \mathrm{~Hz}$ in half number of our subjects independently to the correct or incorrect results. Perhaps all these subjects increase psychic effort independently to results.

However, one must distinguish different psychic categories: concentration (decreasing of alpha activity), mental effort (increasing delta activity), mental level ability (amount of beta activity and changing of alpha pattern) [17-22]. Each person may have a "gift" for various properties.

The EEG spectrum in the first (ALAS) and also in the second half of alpha spindle (ALDE) shows differences, both parts are very similar, optically indiscernible. Usually in ALAS is less of delta and more of alpha amount but during ALDE in contrary i.e. more delta and less alpha [24]. These correct proportionalities were present in 5 people from the group subjects with correct calculation and typically left sided quotients. In group of people with incorrect calculation is never present typical alpha-delta changes in ALAS-ALDE spectrum.

The component delta was increasing and component alpha decreasing during any psychic activity (calculation, Raven test, reading etc.) is more often in healthy subjects. Augmenting delta frequency is perhaps due to mental activity in cortex. The reason for decreasing alpha frequency can be perhaps explained by attention enhancement and hyperactivity in ARAS (ascendant reticular activation system) with "alpha waves typical left sloping" and good quotients (quotus alpha less than $1.0)$.

It was summarized, that the 15 subjects from 22 people alpha waves were not precisely sinusoidal but on the contrary of an asymmetrical shape with steeper and shorter ascendant and longer descendant parts of the waves (see Fig. 1 and Fig. 2). These waves inclined to the left side. Only one woman (BAA) had typical alpha waves inclination to the left side in spite of her non-correct calculation. Remaining 7 subjects have inversely shaped waves, to the right side and 5 subject from them had bad calculation (quotus alpha higher than 1.0). It seems that quotus alpha analysis discriminates better than other methods the healthy and less healthy subjects and simultaneously gives information about augmented and correct mentation. 
Faber J. et al: Dynamics of sinusoidal alpha waves asymmetry in brain...

Children and incorrectly thinking adolescent subjects usually show very often more various biological artifacts in EEG curves and in other signals, e.g. in eyes blinking (electrooculograms, EOG), on muscles movement (electromyogram, EMG), in skin galvanic potentials (SGP) etc., mainly in frontal and temporal regions.

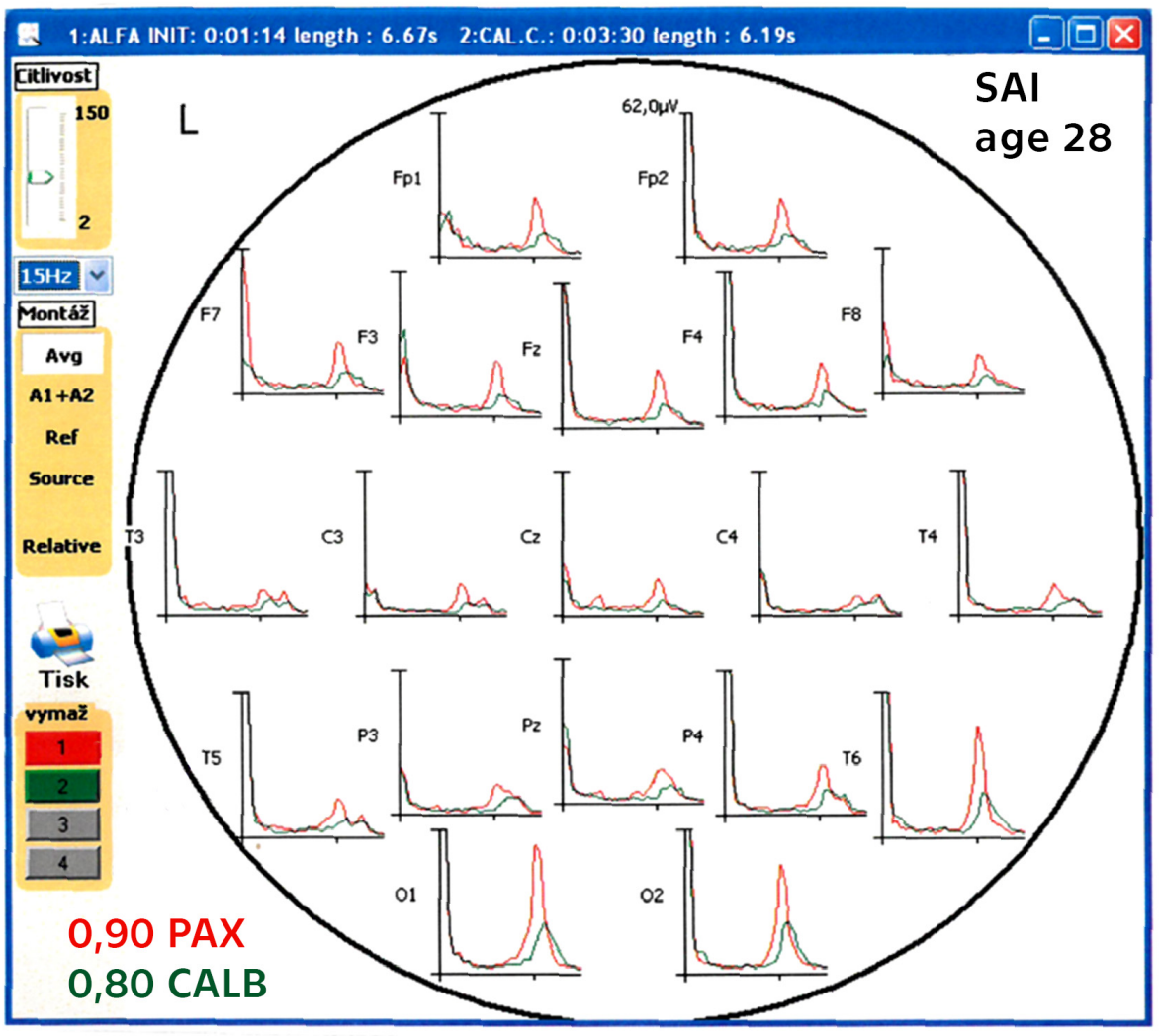

Fig. 3 EEG spectrums according Gabor from all 19 amplifying channels (EEG device by firm DEYMED, Czech Republic) in healthy proband (SAI) aged 28 years with normal EEG curves and correct calculation from young group of 10 perfect thinking subjects. Big letters and numbers (odd on the left side) designate electrode localization, curves determine amount (power) of the frequency band in spectrum (from left to right: delta, theta, alpha and beta), small strokes on x-axis designates $10 \mathrm{~Hz}$, spectrum finished on $15 \mathrm{~Hz}$. Red (higher) curves represent spectrum during PAX, quotus alpha is 0.90, green (lower) curves represents spectrum during correct calculation, quotus alpha is 0.80. Besides there is decrease of slow and increase of faster alpha frequency, lowering of all alpha activity in power spectrum during calculation in comparison with state of relaxation. Alpha has not bimodal feature. In the electrodes $\mathrm{T} 6$ and $\mathrm{O} 2$ there is small delta band increasing during calculation. Seen by naked eyes (optical view only) alpha frequency increases about $1 \mathrm{~Hz}$. Generally speaking, alpha decrease accompanies the subjects' attention increase and delta increase is present during mentation enhancement [7, 16, 22]. 
Neural Network World 6/2019, 427-445

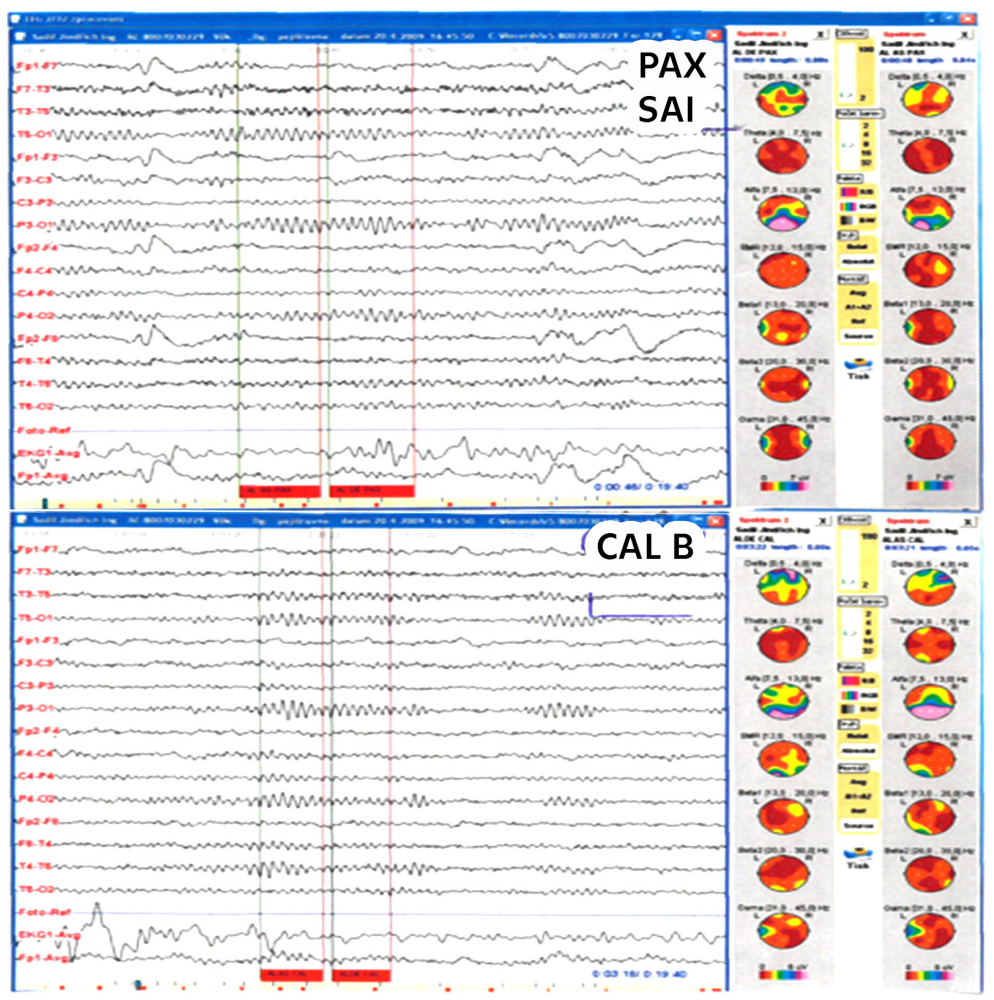

Fig. 4 The same proband (SAI) as in Fig. 3, relaxation state. In the upper part on left side in this figure are 19 channels normal alpha activity in form of fusiform alpha waves. The first color column of brain electrical activity mapping (BEAM) on the right side shows declining spindle with increasing alpha and bifrontally small amount delta power in the second part of alpha spindle (ALDE - alpha descendent). The second BEAM column (totally on the right margin) shows augmenting of recruiting in alpha spindle (ALAS alpha ascendant) with alpha and delta power decreasing. For a wonder these frequency changes are not evident by naked eye. (From up to bottom are color circles representing delta, theta, alpha and 4 beta circles. Color scale shows frequency intensity, the highest is violet, the lowest is beige.) The bottom half of this figure shows the same order as is in upper half, but shows active state during correct calculation. During calculation in ALDE decreases alpha and increases delta power, which is opposite in comparison with ALDE quiet state. During ALAS expressively increases alpha and decreases delta power which is again opposite to ALAS in quiet state. Observing differences ALAS-ALDE in one state and $A L A S-A L D E$ between both states is impossible by simple look, but quite good it is possibly after the spectral filtration [16, 18, 23, 24, 27]. These results are in agreement with reached experiences, described in part Discussion. 
Faber J. et al: Dynamics of sinusoidal alpha waves asymmetry in brain...

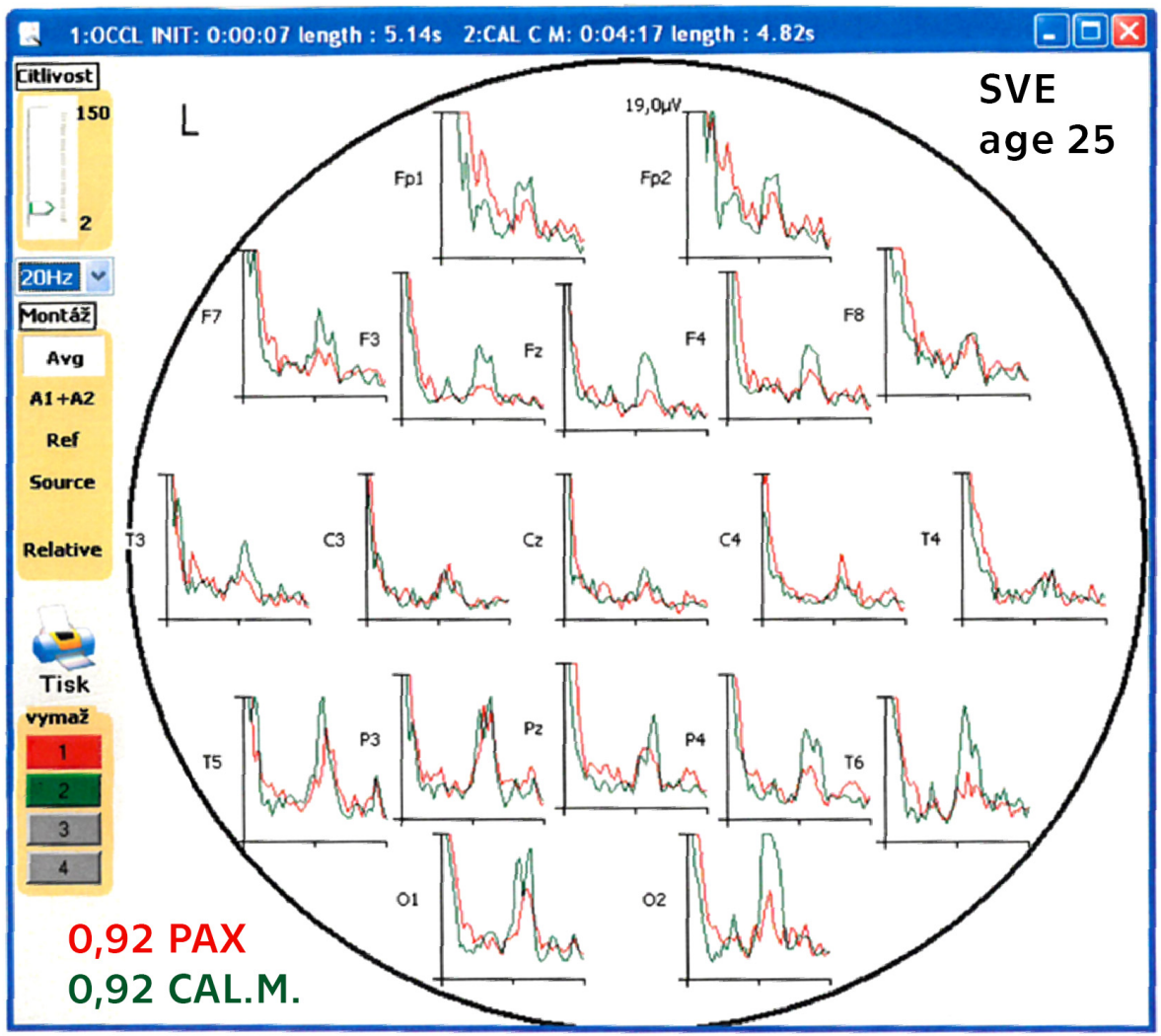

Fig. 5 Man, aged 25 years, (SVE), subjectively without difficulties, normal IQ, (perhaps ADD syndrome), abnormal EEG perhaps post-febricities etiology in childhood, abnormal results in EEG analysis during fault calculation. (Schema and legend is the same like in Fig. 3.). Quotients are high 0.92 during PAX and the same 0.92 during incorrect calculation. Simply said, alpha waves during PAX and mentation is close to 1.0, i.e. their feature does not change during mentation, sinusoidal form alpha waves are still near to symmetry. EEG spectrum shows increasing of rapid, but also slow alpha waves. Paradoxically alpha is higher and delta is lower during calculation than during relaxation. Alpha bimodality is present, more expressive on the left side and mainly in O1, P3 and P4 during calculation. We see the bimodality more often in "neurotic" subjects and generally during intrapsychic tension in anxious syndromes, but during enhancement of mental effort e.g. during psych tests as well. Alpha frequency increases by simple look during calculation about $1 \mathrm{~Hz}$.

Children and non-correct thinking subjects usually shows very often more various biological artifact in EEG curves, e.g. eyes blinking (electrooculogram, EOG), muscles movement (electromyogram, EMG), skin galvanic potentials (SGP, mainly in frontal and temporal regions), sometimes accelerated heart rate, etc. These results remind experiences with lie detector, although the distinguishing of liars and neurotic subjects by help of these changes may be difficult. 


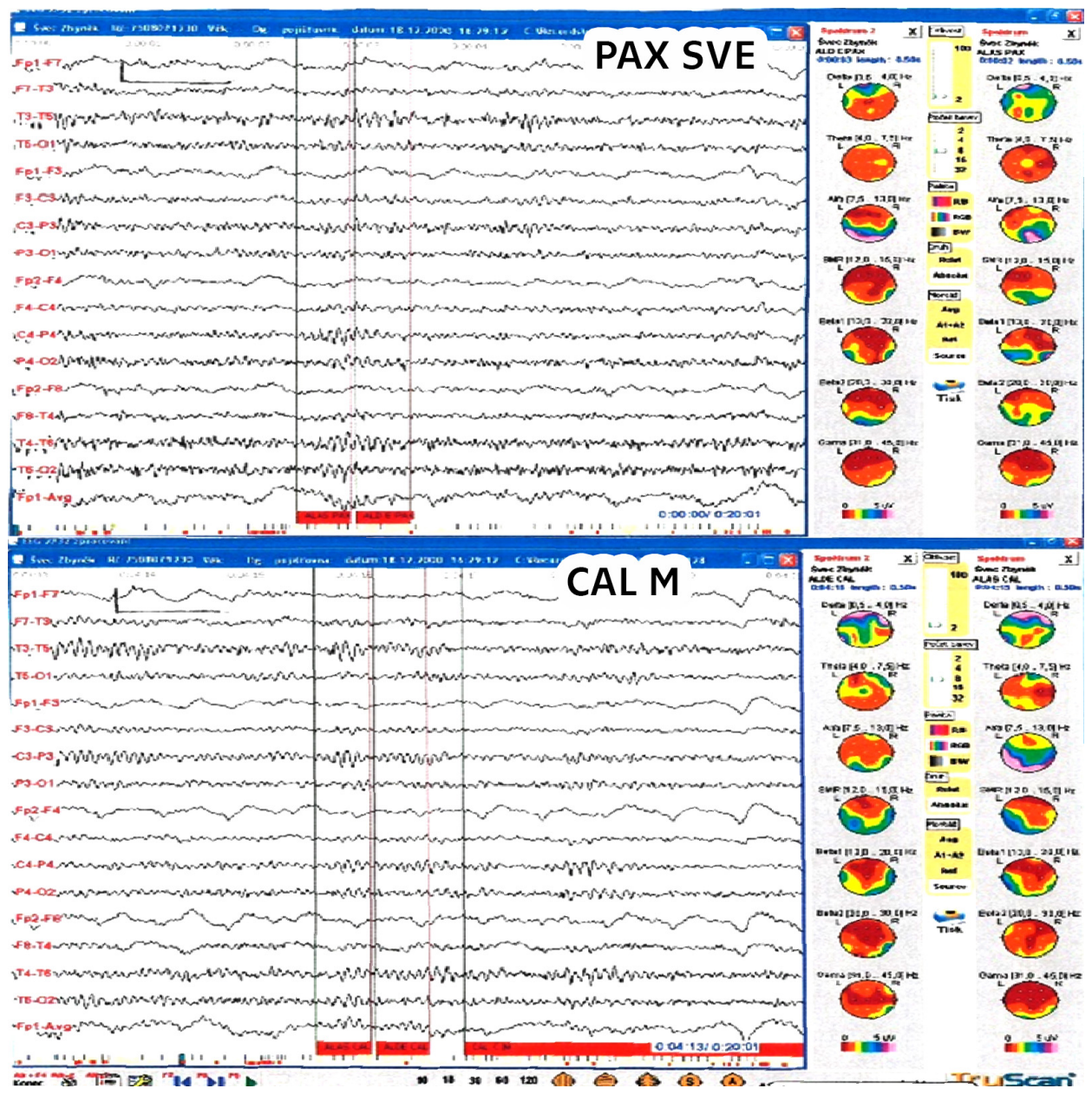

Fig. 6 EEG curves and results of BEAM in the same proband (SVE) from previous Fig. 5. (The order and legend is the same as in Fig. 4.). EEG curve in PAX shows irregular fusiform alpha wave with high amplitude. ALAS has relatively higher amount of delta activity frontally left and more beta activity occipital left than in ALDE and less of alpha activity occipital in ALAS than in ALDE. During calculation with mistakes has delta activity in ALAS almost the same amount as in ALDE bi-frontally a more alpha and beta activities on the left side temporo-occipital in ALAS than in ALDE. Gabor spectral analysis shows similar results in both probands (SAI and SVE), but SVE has substantially more beta activity than SAI. Optically seen alpha activity increases about $1 \mathrm{~Hz}$ during calculation. 
Faber J. et al: Dynamics of sinusoidal alpha waves asymmetry in brain...

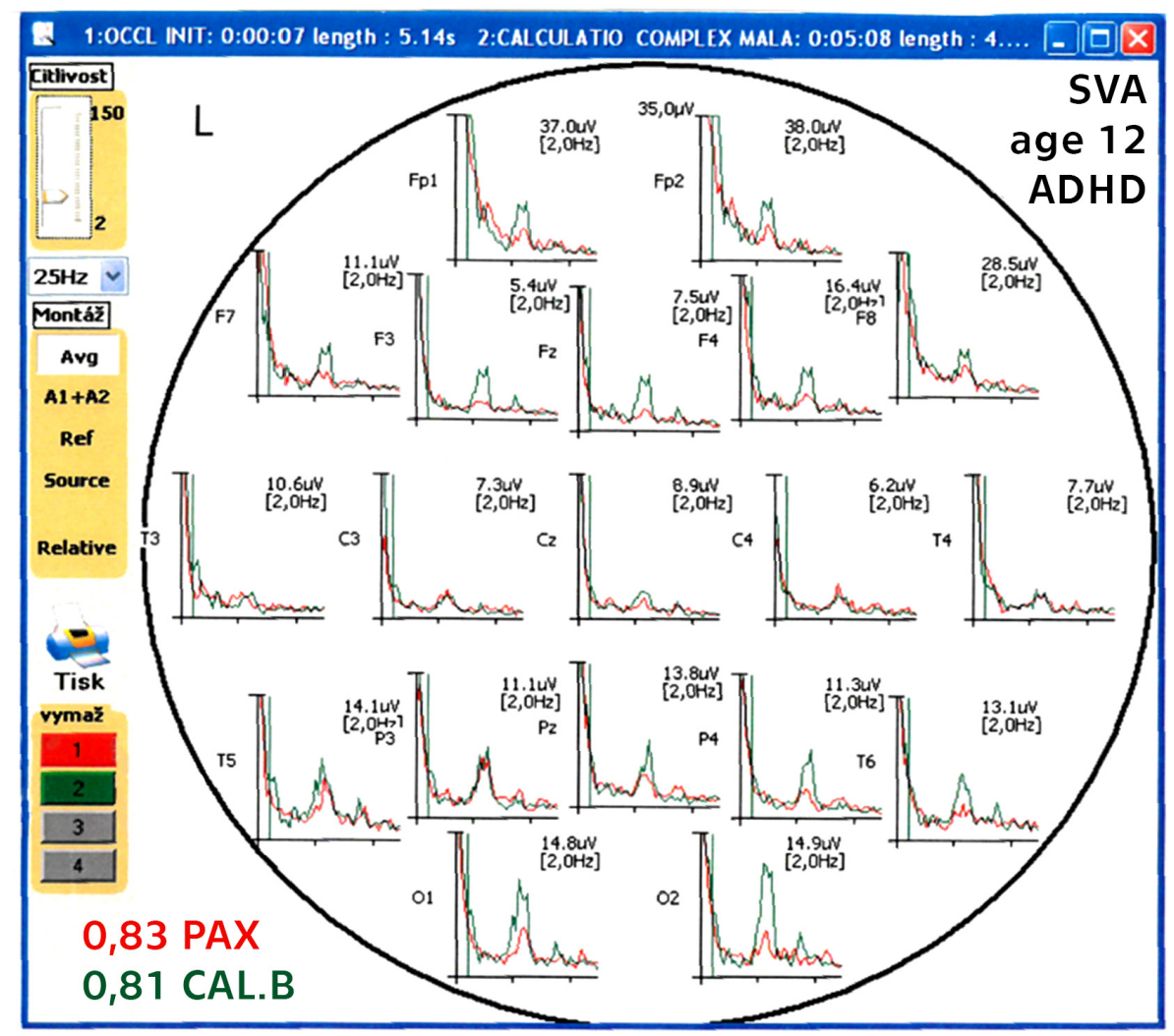

Fig. 7 (The order and legend is the same as in Fig. 3.) EEG spectrum (sec. Gabor), boy, aged 12 years (SVA), he suffered from ADHD, good calculation results. Abnormal EEG curve, typical for this syndrome: slow irregular non-fusiform alpha or pre-alpha activity with high amplitude, often occur episodes of high slow sharp alpha waves followed by slow theta or delta waves of irregular shape with prevalence over the temporo-parieto-occipital region. Quotient (the ratio between ascendant and descendent parts of alpha waves quotus alpha) during PAX is 0.83, and during calculation is 0.81. The difference is small, but typical alpha waves have small inclination to the left side and during calculation this sloping increases. Alpha activity behaves are bilaterally different, over O2 and P4 are accelerated about half of $\mathrm{Hz}$ and over T5 and $\mathrm{O} 1$ slow down by $1 \mathrm{~Hz}$. Over T5, O1 and T3 electrodes increases a little delta activity. Alpha activity amplitude paradoxically increases, shape is changed to dichotomy and creased beta $17 \mathrm{~Hz}$ in temporo-occipital region during calculation. Calculation is correct in case of this boy with good IQ, but thanks to ADHD syndrome are quiet and mentally stimulated EEG results partly typical, partly very atypical.

\section{Discussion}

The first used proband group contained 22 persons: 10 healthy subjects, 7 children suffered from ADHD, 1 epileptics had typical epileptic graph elements, 4 subjects suffered from neurotic symptoms. 15 subjects had abnormal EEG curves (diffusely 


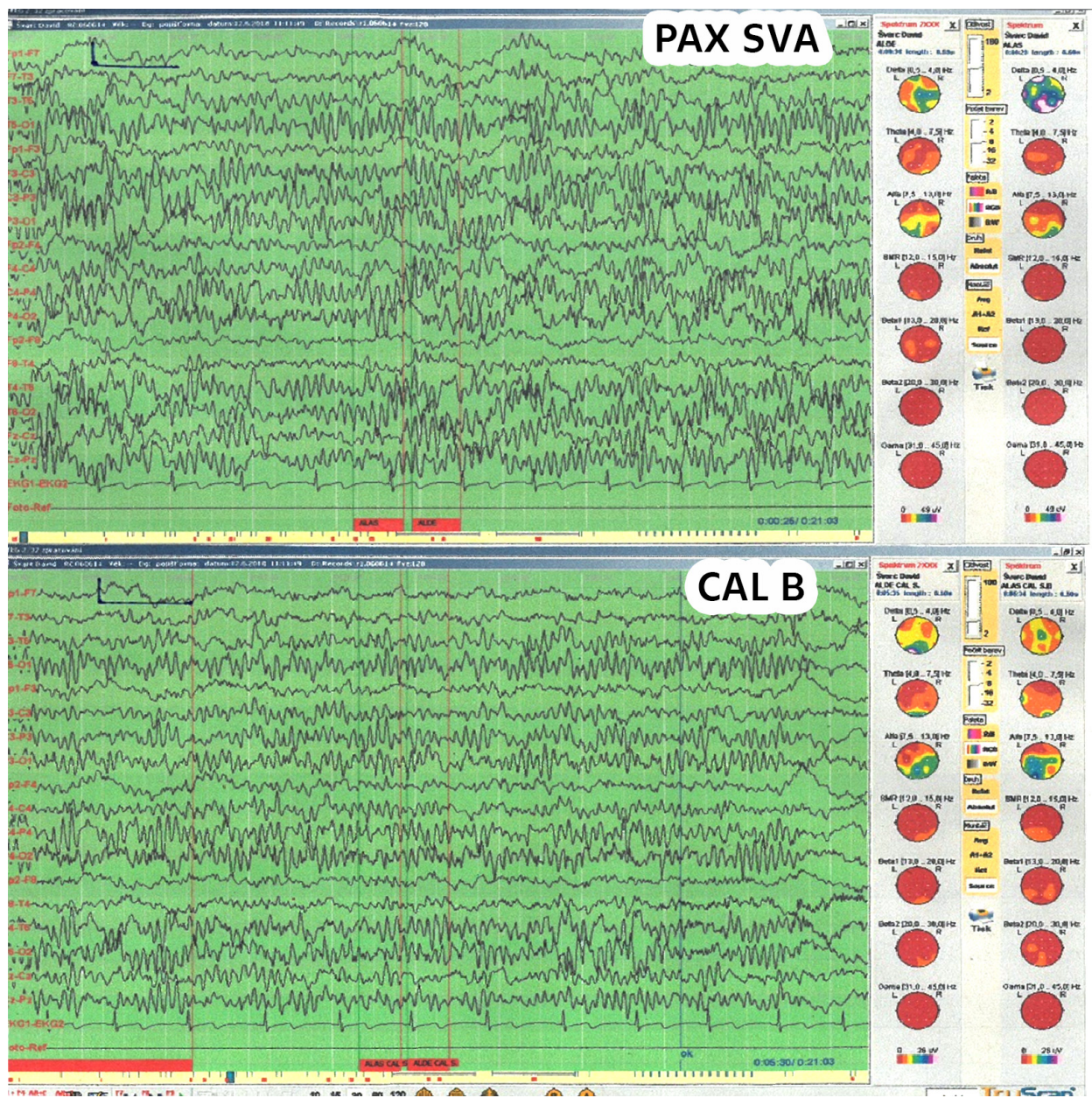

Fig. 8 EEG curves and EEG analysis results in previous boy SVA (Fig. 7). (The order and legend is the same as in Fig. 6 and the Fig. 4.) The EEG is typical for ADHD syndrome (see Fig. 7). BEAM shows in quiet state in ALAS higher power of delta activity which is atypically localized occipitally and less also frontally on the left side and relatively less alpha power in occipital region in the right side. Beta activity amount is minimal. Sub-delta activity is perhaps due to artifact, e.g. skin galvanic potentials (SGP). During calculation, minimal delta activity is present in $A L A S$ over $C z$ electrodes but enhancement of alpha activity occipitally left, beta frequency is again very low. Sub-delta band is substantially depressed in comparison with quiet BEAM. During calculation is alpha higher than during ALDE and is localized occipitally on the right side. During PAX is prevalence alpha on the left side, which is atypical for normal state but typical for ADHD syndrome [24]. 
Faber J. et al: Dynamics of sinusoidal alpha waves asymmetry in brain...

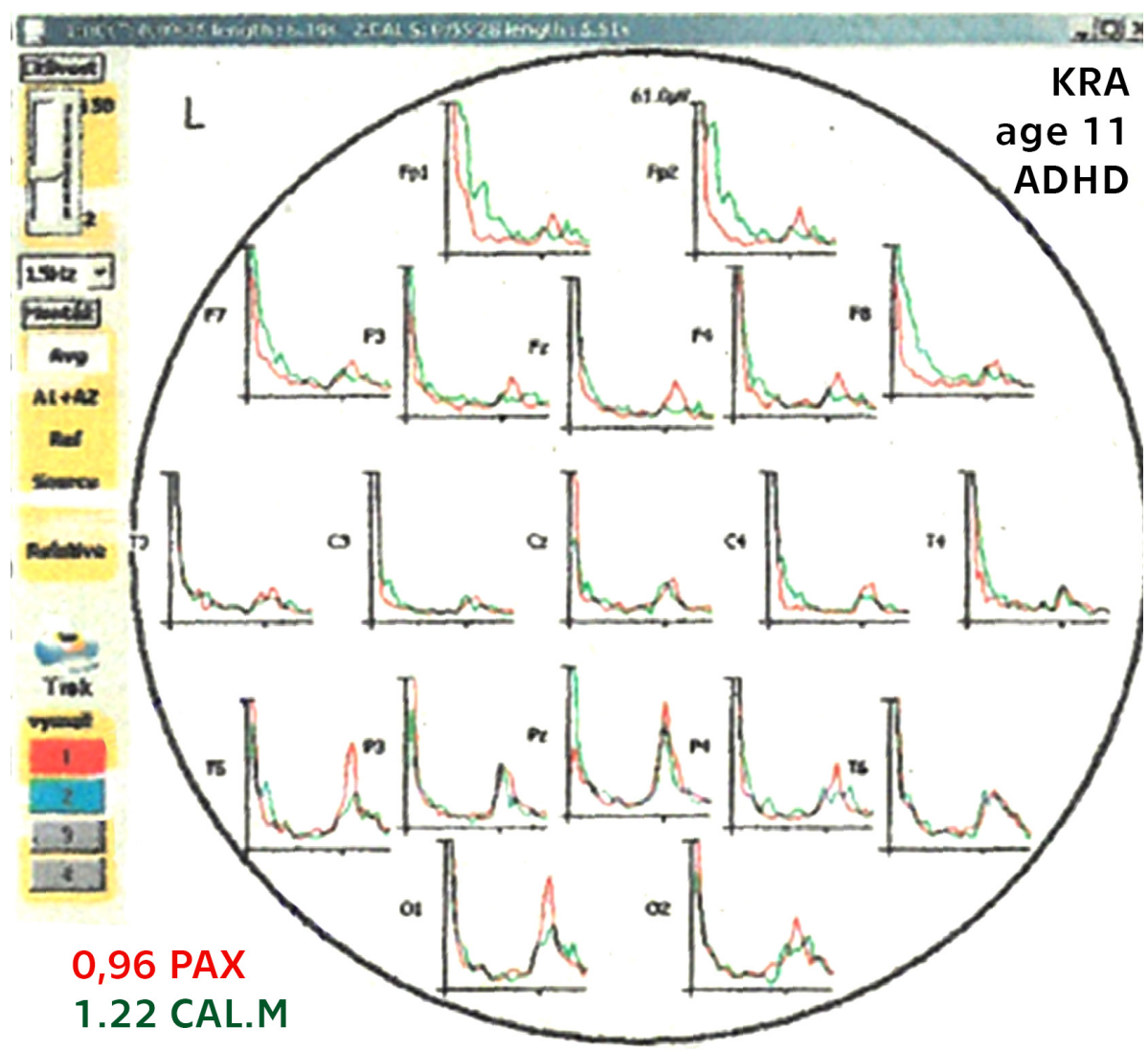

Fig. 9 EEG spectrum, girl KRA, aged 11 years, ADHD syndrome, fault in calculation, EEG is abnormal, alpha frequency paradoxically decreased during calculation from 12 to $11 \mathrm{~Hz}$. (Order and legend is the same as in Fig. 3, 5, 7.) During quiet state (red curves) dominates delta and alpha (12 Hz) bands of high amplitude mainly in T5, Pz, and $\mathrm{O1}$ regions. During calculation (green curves) is small alpha acceleration in $\mathrm{P} 4, \mathrm{O} 1$, and O2 regions and increases delta amount in $C 3, C z, C 4, T 5, P z$ and $P 4$, alpha activity paradoxically slows down in $C z, P 3$ and P4. Asymmetry of quotient (quotus alpha), in PAX equals to 0.96, therefore almost symmetrical are sinusoidal alpha waves. During calculation with mistakes is quotient 1.22, therefore inversely oriented alpha curves, because of expressive inclination to the right side. During calculation is present alpha dichotomy, which was not present during quiet state. These atypical and abnormal results partially explain faults and attention disturbances during mental activity.

or episodically theta or delta is present). 7 probands had normal EEG with alpha activity only, short neurological and psychological investigation was in normal limits. Provided new analysis of quotus alpha show for 11 subjects during PAX quotient less than 1 (quotus alpha of average 0.81 ) and further lower during correct calculation (quotus alpha of average 0.73) (one exception with incorrect calculation, subject BAA). Next 11 subjects: 4 times non correct calculation) quotient during 


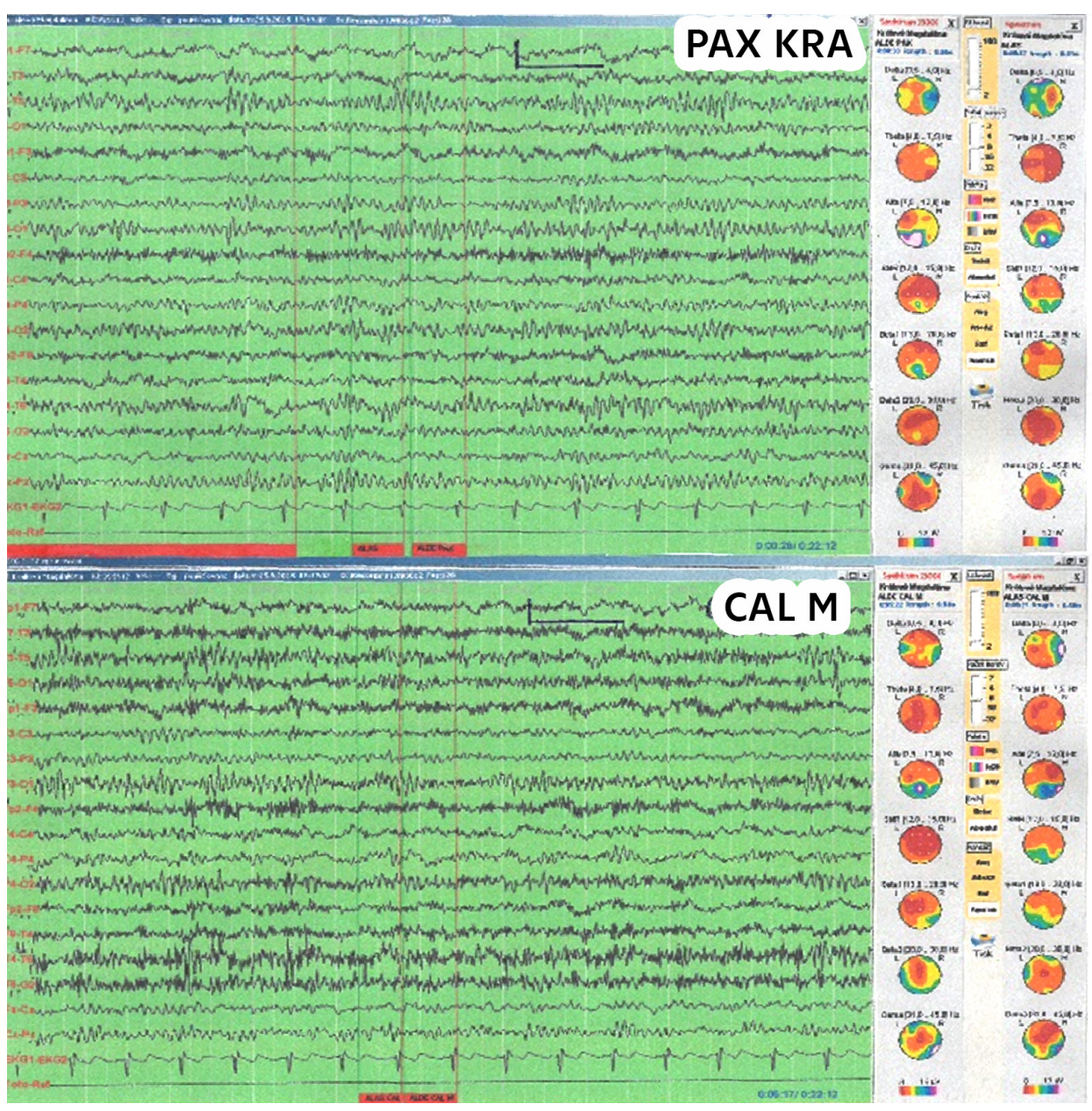

Fig. 10 EEG curves, the same girl KRA as above in Fig. 9. EEG curves are abnormal because of diffuse theta waves and very irregular alpha waves which are not typical for ADHD syndrome (Order and legend is the same as in Fig. 4, 6 and 8.). Optically it is seen the decrease of alpha amplitude during calculation (bottom half of the figure) as a manifestation of attention enhancement. ALAS and its BEAM shows in quiet state delta increasing over the left hemisphere and adequate alpha amount occipitally. ALDE has less delta power amount than in $A L A S$ with clear prevalence over the right hemisphere and increasing alpha amount occipitaly on the left side. Slow beta (12-15 Hz) increases in $A L A S$, in ALDE is faster beta (13-20 Hz) also occipitaly. BEAM during calculation shows focal prevalence delta temporally in the right side and alpha bi-occipitaly. Beta band in ALAS and less in ALDE is expressive but we ignore them because of muscles artifacts good perceptible in EEG curves, evidently produced by increasing intrapsychical tension during psychological test. ECG curve is during the testing faster than in quiet state as well. 
Faber J. et al: Dynamics of sinusoidal alpha waves asymmetry in brain...

PAX is 1 or higher than 1 ( 5 times) or inverted quotient (6 times) (quotus alpha on an average 0.89) and quotients during calculation is atypically higher than during PAX (i.e. inversion) (on an average 0.98). Perhaps the left sloping alpha waves are more advanced for mental processes in TCRIS (thalamo-cortical reverberation and iteration system). Quotus alpha results are changing not exactly dependent to optically described normal or abnormal EEG curve. Also not to alpha frequency acceleration or slow down. One sees again very complex brain system creating complicated trajectory and no analysis is able explain this complexity. In addition, one must in EEG waves or in EEG spectrum admit not only regular components, but also irregular, i.e. random variables. Of course, noise is really present in working brain, it was confirmed by use of Wilks periodogram [18, 19, 33, 37].

Some authors speculate about the communication problem not only with neuronal cells but also with giant number of all active synapses. But one must not forgot that thanks to THCRIS one do not move in completely unknown order less system [24-27] but also in genetically preformed space.

Alpha waves are mostly asymmetric, as it is visible as little slope to left side. The ascendant part of alpha wave is shorter and steeper than the descendent one. During successfully mental activity e.g. correct calculation, is even more steep. However, usually during non-correct calculation is the proportionality in majority of cases inverted, i.e. the ascendant alpha wave part is longer than the descendent one. This shape changes quickly and often, these alternations are depending on the states of concentration, vigilance, mood, IQ etc. Therefore, we proposed new analytical method (quotus alpha) which allows describe this variable EEG graph element and mental process (psychical mechanism in TCRIS) by help of one number only. Quotient less than 1.0 shows good and correct actual mental activity, higher quotient than 1.0 usually represents mental activity with mistakes.

Good reckoners had decreasing alpha amount, increasing alpha frequency, a little increasing delta in EEG spectrum, moreover remains during calculation alpha waves spectral mono-modality. However, mistaken subjects had contrary results and high variability in tests results. Alpha wave steep shape is perhaps due to different (PAX or CAL.) recruiting quality and intensity. Let imagine, that is higher and faster the alpha ascendant, the higher is amount of neuronal impulses in sequences and more non-exact information, rolling from thalamus to cortex. These impulses are generally more synchronized during ALAS, than in ALDE and more during ALAS in mentation than in quiet state. Simultaneously amplitude alpha and alpha spectral amount decreases thanks to attention enhancement due to influence of the ARAS (ascendant reticular activation system). During descendent alpha wave stage increases "optically" seen beta activity and "spectral" seen delta activity. Already Berger [7] speculate about beta rhythm as physiologically hyperactive function. EEG spectral analysis during decrescent alpha spindle (ALDE) shows evident increasing delta frequency band. Perhaps thanks to second half in alpha spindle (ALDE) and descendent trend of alpha wave, the flowing impulses back to thalamus after cortical data processing have less amount, but are selected and less synchronized, more specific to some neurons. Cortical data processing (mainly during ALDE) is perhaps specific selective impulse activity producing more or less competent neurons. But these neuronal nets are instable, therefore must be this process often and many time repeated in TCRIS. 
Simultaneously starts mainly during more difficult psych tests the subcortical association and commissural system (SACS) which produces slower frequencies (delta and theta) [18-20,25-28,32,35]. Young subjects aged up to 15-20 years calculate one digit numbers (CAL.S.) usually with alpha frequency acceleration, but two digit numbers (CAL.C.). shows moreover delta enhancement in young and adolescents as well. One proposes this interpretation: manipulation in CAL.S. represents more simple mental process implemented in THCRIS by help of alpha frequency. Mentation during CAL.C. is more complex process which is realized not only in THCRIS but also in subcortical association and commissural system (SACS), where the data processing is more time consuming [22,24]. Thanks to spectral delta increasing one can recognize, that the proband is thinking and now one also knows by help of quotus alpha, if he is thinking correct or incorrect.

Alpha waves have many intriguing properties thanks to TCRIS, e.g. functions of Daedaleum Kinesiskop according to J. E. Purkyně $[26,27,30]$, which have symptoms of fractal trajectory and ability manipulate usefully with random and harmonic data together or to have very similar recruiting parameters in "healthy" vigilance as in pathological states, e.g. in epileptic absences with $\mathrm{S}+\mathrm{W}$ graph elements in EEG or in dissociative states $[1,9,21,24,29]$ occurring in the same subject [17].

\section{Conclusion}

Changes in shape of alpha waves in EEG (electroencephalogram) were observed with use of presented method, which compare and calculate of ascendant and descendant parts of alpha wave in EEG, one can call it quotus alpha. Let us say that alpha wave is similar to nonrectangular triangle with asymmetrical legs. Legs or vector ratio shows the grade and sense of asymmetry. The results varies between $0.60-0.90$, which means that the alpha wave is inclined to the left side (ALIL) and 1.0-1.30, which means that the alpha wave is inclined to the right side (ALIR). Only sporadically are the alpha waves symmetrical.

The used proband group had 22 subjects, aged 7-34 years, 18 men, 4 women who were investigated by help of Raven test and EEG during two states: PAX (OCCL, quiet state, relaxation) and mental activity CAL.S. calculation (addition of one digit numbers $\mathrm{S}=$ simplex or addition of two digit numbers $\mathrm{C}=$ complex, B (Bona, correct) or M (Mala, incorrect), all persons and states with eyes closed. 14 adolescent peoples had ALIL, therefore quotus alpha less than 1.0. Six subjects aged 7 up 18 years suffered from ADHD with typical abnormal EEG had 5 times alpha waves ALIR type (quotus alpha higher than 1.0), together with dichotomy alpha waves during incorrect ( 3 times) calculation. 10 healthy peoples aged 22 up to 34 years had all the time ALIL (quotus alpha less than 1.0), higher inclination to the left during calculation, which was in this subjects still correct with increasing alpha frequency about $1 \mathrm{~Hz}$. EEG spectrum shows during calculation decreasing slow and increasing rapid alpha frequency and increasing delta spectral amount. Remaining 5 adolescent subjects had incorrect calculation, ALIR alpha organization (quotus alpha higher than 1.0) and subjectively feeling neurotic symptoms.

Generally spoken, one can say that the delta activity increase means the stronger thinking, but it seems also that quotus alpha ALIL type represent successful solution of considered problem. 
Faber J. et al: Dynamics of sinusoidal alpha waves asymmetry in brain...

Of course, the certification of this statement as increase the probability in the field of many uncertain factors will need to provide much more measurements of much larger groups of probands. A wider applicability of the knowledge of here discussed brain dynamics could have use also in wider fields and real applications, like those mentioned in $[38,39]$ or [41]. Of special interest can be also the projection of the here discussed results in the physics/information analogies (see e.g. [40,42]).

Nevertheless, such further results, which are expected to be reached in the course of continuation of this research, which is hoped to start with not too large delay, can be of really high value, especially if their correspondence with the previous facts will be certified.

\section{References}

[1] ANAND B.K., CHHINA G.S., SINGH B. Some aspects of electroencephalographic studies in Yogis, Electroencephalography and Clinical Neurophysiology, 13(3), 1961, pp. 452-456, ISSN 0013-4694, doi: 10.1016/0013-4694(61)90015-3.

[2] ANDERSEN P., ANDERSSON S. A. The alamiíc Origin of Cortical Rhythmic Activity. PP 2C-00-118. In: Handbook of EEG. Clin. Neurophysiol. Ed. D. Creutzfeldt, 1974, 2.

[3] ASHBY W.R.: Kybernetika, MME, Orbis, Praha, 1956, (in Czech).

[4] AtKinsonová R.L., ATKinson R.C., SMith E.E., BEM D.J. Psychologie. Victoria Publishing, Prague, 1995, (in Czech).

[5] BATTAGLiA F.P., SUTHERLAND G.R., COWEN S.L. Firing rate modulation: A simple statistical view of memory trace reactivation. Neural Network, 2005, 8, pp. 1280-1291, doi: $10.1016 / j$.neunet. 2005.08.011.

[6] BENEŠ J. The cybernetic systems with automatic organization. Academia, Praha, 1966, (in Czech).

[7] BERGER H. Das Elektrenkephalogram des Menschen. Nova Acta Leopoldina. Halle, Saale, (1938).

[8] BINSWANGER L. Grundformen und Erkentnis des menschlichen Daseins. München, 1964. Bleuler E: Lehrbuch der Psychiatrie, Springer, Berlin, 1930.

[9] BOB P., FABER J. Quantum information in brain neuronal network and electroencephalogram. Neural Network World, 1999, Pragure, 4, pp. 326-372.

[10] BOROVANSKÝ L. Soustavná anatomie. (Systematic Anatomy). Díl VII. Neuroanatomy. ZN, Praha, 1951, (in Czech).

[11] BOUCHAL M., KONEČNÝ R. Psychologie v lékařství. (Psycholgy in Medicine), SZN, Praha, 1966, (in Czech).

[12] BOWLBY J. Secure base. Tavistok, Routledge, London, 1995.

[13] BRÁZDIL M., REKTOR I., DUFEK M., JURÁK P., DANIEL P.: Effect of subthresold stimuli on even- related potentials. Electroenceph. Clin. Neurophysiol., 1998, 10, pp. 764768.

[14] CARLSON A., WATERS N., CARLSSON M.L. Neurotransmitter Interaction in Schizophrenia, Therapeutic Implications. Biol Psychiatry, 1999, 46, pp. 1388-1395, doi: 10.1016/ S0006-3223(99) 00117-1.

[15] BOUCHNER P., FABER J., NOVOTNÝ S., TICHÝ T. Drivers attention level improvement with use of biofeedback stimulation incorporated into driving stimuilator. Neural Network World, 1999, 09, pp. 109-118.

[16] DOLCE G., WALDEIER H. Spectral and multivariate analysis of EEG changes during mental activity in man. Electroenceph. Clin. Neurophysiol., 1974, Prague, 36, pp. 577-584, doi: https://doi.org/10.1016/0013-4694(74)90224-7. 


\section{Neural Network World 6/2019, 427-445}

[17] FABER J. Formal Aspects of Vigilance during Petit Mal Paroxysm. Acta Universitatis Carolinae Medica. Monogr. LXIV. Universitas Carolinae, Prague. 1975.

[18] FABER J. Vigilance, Sleep, Petit Mal and Electroencephalogram as manifestations of programmed brain regulation. Acta Universitatis Carolinae Medica, Monogr. LXXXVII. Universita Karlova Praha. 1978.

[19] FABER J. Associative Interneuronal Biological Mechanism.Neural Network World 1991, l, pp. $13-31$.

[20] FABER J. Electroencephalography and psychophysiology, ISV Pub. house, Prague, 2001, pp. 170, (in Czech).

[21] FABER J. Isagogé to non-linear dynamics of formators and complexes in the CNS. Acta Universitatis Carolinae Medica, Monogr. CXLIX, Praha 2003.

[22] FABER J. QEEG, Galén, Prague, 2005.

[23] FABER J., PILAŘOVÁ M., VUČKOVÁ Z., BÖHMOVÁ D., DOBOŠOVÁ L.: Praktická aplikace EEG analýsy i neurologických a psychiatrických chorob. (Practical application of EEG analysis in neurological and psychiatric diseases.) Prakt. Lék., 1998, 98, pp. 227-233. (In Czech).

[24] FABER J. Bulbo-thalamo-cortical structures and thein Activities Stimulated by "FormatorComplex" and "Multilayered Iterative Algorithm" Models, Acta Universitatis Carolinae Medica, Monogr. CLX. Charles University in Prague, 2015.

[25] FABER J., SADIL J., KOVALJOV M. Cognitive functions of biological and artificial network. I. M. Marci, Prague, 2017, a.

[26] FABER J., SADIL J., KOVALJOV M. The digitalized psychic. I. M. Marci, Prague, 2017.

[27] FABER J. Intrapsychická imaginace je iluzí digitalizované mentace. (Intrapsychical imagination is illusion of digitalized mentation.) I. M. Marci, Prague, 2018. (In Czech).

[28] IVAKHNENKO A.G. Heuristic Self-Organization in Problems of Enginearing Cybernetics. Automatica. 1970, 6, pp. 207-219, doi: 10.1016/0005-1098(70)90092-0.

[29] JANET P. The psycholeptic crises. Boston Medical and Surgical Journal, 1905, CLII, 4, pp. 93-100, doi: 10.1056/NEJM190501261520401.

[30] KRUTA V.: Jan Evagelista Purkyně (1787-1869). Centenary Symposium. Universita J. E. Purkyně, 1971.

[31] LION K.S., WINTER D.F.: A method for discrimination between signal random noise of electrobiological potentials. Electroenceph. clin. Neurophysiol. 1953, 5, pp. 109-117, doi: 10. 1016/0013-4694(53) 90060-1.

[32] NOVÁK M.: Umělé neuronové sítě, teorie a praxe. (The artificial neuronal network, theory and praxis). C.H. Beck Prague, (1998). (In Czech).

[33] NOVÁK M., VOTRUBA Z., FABER J.: Impacts of driver attention faillures on transport reliability and safety and possibilities of its minimizing. Neural Network World, Prague, 2004, 1/4, pp. 49-56.

[34] RICCI G., DOANE B., JASPER H. Microelectrode of conditioning: technice and preliminary results. Premier congrés international des science neurologique. Acta Medica Belgica, Bruxelles, 1957.

[35] ŠNOREK M. Neuronové sítě a nuropočítače. (Neuronal Nets and Neurocomputers). Prague, ČVUT, Czech Technical University, 2004 (In Czech).

[36] SAUNDERS M.G.: Amplitude probability density studies on alpha and alpha-like patterns. Electroenceph. clin. Neurophysiol., 1963, 15, pp. 761-769, doi: 10.1016/0013-4694 (63) 90166-4.

[37] WILKS S.S.: Mathematical Statistics. John Willey, New York, London, 1962.

[38] KAMINOZHISELVI C.S., PRATAP A.: Possibilistic LQV neural network - an application tp chldhood autism grading, $N N W, 2016,26$, pp. 271-287.

[39] SUNG HO JANG, HYUEOG GUY KWON Connectivity of inferior cerebellar peduncle in the human brain: a diffusion tensor imaging study, $N N W$, 2016, 26, pp. 439-447. 
Faber J. et al: Dynamics of sinusoidal alpha waves asymmetry in brain...

[40] SVITEK M.: Physics - information analogies, $N N W$, 2018, 28, pp. 535-550.

[41] GARLÍK B.: Application of neural networks and evolutionary algorithms to solve energy optimization and unit commitment for a smart city, $N N W, 2018,28$, pp. 379-413.

[42] MOOS P., SVÍTEK M., NOVÁK M., VOTRUBA Z.: Information model of resonance phenomena in brain neural networks, $N N W, 2018,28$, pp. 225-239. 